OPEN ACCESS

Edited by:

Ge Zhang,

Hong Kong Baptist University, China

Reviewed by:

Ichiro Hirao,

Institute of Bioengineering and Nanotechnology (A*STAR),

Singapore

Zhiling Yu,

Hong Kong Baptist University, China

*Correspondence:

Zhenjun Yang

yangzj@bjmu.edu.cn

tThese authors have contributed equally to this work and share first

authorship

Specialty section:

This article was submitted to

Molecular Medicine

a section of the journal

Frontiers in Cell and Developmental

Biology

Received: 29 January 2021 Accepted: 01 June 2021

Published: 28 June 2021

Citation:

Wu J, Wang S, Li X, Zhang $Q$, Yang J, Ma Y, Guan Z and Yang Z (2021) Selective Anti-melanoma Effect of Phosphothioated Aptamer Encapsulated by Neutral

Cytidiny//Cationic Lipids.

Front. Cell Dev. Biol. 9:660233. doi: 10.3389/fcell.2021.660233

\section{Selective Anti-melanoma Effect of Phosphothioated Aptamer Encapsulated by Neutral Cytidinyl/Cationic Lipids}

\author{
Jing Wut, Shuhe Wang ${ }^{\dagger}$, Xiang Li, Qi Zhang, Jie Yang, Yuan Ma, Zhu Guan and \\ Zhenjun Yang*
}

State Key Laboratory of Natural and Biomimetic Drugs, School of Pharmaceutical Sciences, Peking University, Beijing, China

BC15-31 is a DNA aptamer that targets heterogeneous nuclear ribonucleoprotein A1 (hnRNP A1), which plays a crucial role in the process of pre-RNA maturation and is also essential for the rapid proliferation of tumor cells. In this research, we modified BC15-31 with a phosphorothioate (PS) backbone, LNA, and 2-O-MOE to enhance its stability and target affinity. In addition, a neutral cytidinyl lipid (DNCA) and a cationic lipid (CLD) were mixed to encapsulate modified aptamers with the aim of improving their cell permeability with low toxicity. Under the DNCA/CLD package, aptamers are mainly distributed in the nucleus. A modified sequence WW-24 showed an excellent selective anti-melanoma (A375 cells, 25 nM, 80\%) activity, targeted to both hnRNP A1 and hnRNP A2/B1 found by the BLI experiment, and induced more early and late apoptosis in vitro, which also showed stronger antitumor effect and longer accumulation time in vivo. These results provide a new strategy for further clinical applications.

Keywords: aptamer, phosphorothioate, cytidinyl lipid, hnRNP A2/B1, Liposome

\section{INTRODUCTION}

Aptamers, which Szostak and Ellington first reported in 1990, are single-stranded oligonucleotides or peptides. The oligonucleotide aptamer can fold into a stable 3D structure through intramolecular base interactions in order to specifically bind to its target, which could be a metal ion, a small molecular compound, a nucleic acid, or a protein (Mascini et al., 2012); this binding occurs through $\pi$-stacking, H-binding, and/or electrostatic interaction (Hermann and Patel, 2000). The classic structures of oligonucleotide aptamers predominantly include stem loops (Tok et al., 2000), internal loops, pseudoknots (Park et al., 2008), kissing complexes (Chovelon et al., 2018), hairpin structures (Wen et al., 2016), and G-quadruplex structures (Yang et al., 2017; Zhou et al., 2018). Compared with antibodies, aptamers have multiple advantages: (1) the screening process of aptamers can be performed faster and easier in vitro, (2) the synthesis of aptamers is easy to control and not easily contaminated by bacteria and viruses, (3) aptamers have low impacts on immunogenicity, (4) the thermal stability of aptamers is better than that of antibodies (for example, a DNA aptamer can be 
stored for a long time at room temperature), and (5) aptamers are easy to be modified and can be covalently conjugated with fluorescent groups or other multifunctional groups for disease diagnosis or biochemical detection. Although aptamers have many advantages, they are rarely used as drugs for systemic administration because of their high electronegativity, hydrophilicity, and high molecular weight as oligonucleotides, making it challenging to penetrate cell membranes (Hoerter and Walter, 2007). Moreover, the half-life of naked nucleic acid in serum is about $15 \mathrm{~min}$ due to the degradation of the nuclease that is widely present in the blood, skin, and other organs (Sahay et al., 2010). The only aptamer-based drug approved by the FDA occurred in 2004, which was for the treatment of neovascular (wet) age-related macular degeneration (AMD). Using a local injection of high doses of pegaptanib sodium overcomes the problems above. The cationic liposomes are commonly used to protect the oligonucleotides in vivo and to improve their trans-membrane ability to achieve systemic administration. Nevertheless, the toxicity of cationic liposomes as nucleic acid drug carriers in vivo remains one of the obstacles (Filion and Phillips, 1997; Knudsen et al., 2015; Cui et al., 2018).

Heterogeneous nuclear ribonucleoproteins A1 (hnRNP A1) and A2/B1 (hnRNP A2/B1) are the most important members of the family. Both of them are often found overexpressed in many tumor cells (Pino et al., 2003; Ushigome et al., 2005). Because of its RNA-binding motifs (Xu et al., 1997; Ding et al., 1999), hnRNP A1 is also able to bind to G-quadruplexes or G-rich telomeric DNA (Xodo et al., 2008; Neriec and Percipalle, 2018) and plays an important role in stimulating telomerase activity (Zhang et al., 2006) and the maturation of pre-mRNA (Burd and Dreyfuss, 1994; He et al., 2005). The hnRNP A2/B1 is related to the occurrence and development of apoptosis inhibition and the invasion of tumor cells. Downregulation of hnRNP A1 or hnRNP A2/B1 by siRNA can decrease the invasion and migration of tumor cells, thus hinting that they are important for some tumor-related processes (Li et al., 2012; Dowling et al., 2015). hnRNP A2/B1 is also crucial for gene expression and mRNA stability and participates in controlling the protein translation (Zhang et al., 2017).

BC15 (74 nt) is a DNA aptamer that was found by tissue slide-based SELEX, which has a high affinity to hnRNP A1 and A2/B1 (Li et al., 2009), showing better antiproliferation activity in the HepG2 cell line ( $\mathrm{Ku}$ et al., 2015). We found that the core sequence of BC15, BC15-31 (31 nt), showed higher target affinity and further enhanced bioactivity and stability with isothymidine (isoT) modification at $5^{\prime}$ - and $3^{\prime}$-terminal regions ( $\mathrm{Li}$ et al., 2018). Application of neutral cytidinyl lipid DNCA, through H-binding and $\pi-\pi$ interaction, to reduce or avoid the use of cystine-based cationic lipid CLD (Ma et al., 2016), gave a safe and effective delivery system for oligonucleotides (Ma et al., 2018, 2019). In this study, phosphorathiolation (PS), LNA (ribose locked by a $\mathrm{O}-2^{\prime}-\mathrm{C} 4^{\prime}$-methylene linkage), and $2^{\prime}$-methoxyethoxy $\left(2^{\prime}-\mathrm{O}\right.$-MOE) modification strategies were used to improve the stability and target affinity of the aptamer BC15-31. With the mixture of DNCA and CLD as a carrier, a modified aptamer WW$24(25 \mathrm{nM})$ showed higher target affinity and serum stability, which entered the nucleus, exerting a selective anti-melanoma activity, which could target hnRNP A1 and A2/B1. It also showed better antitumor effect and longer accumulation time in vivo.

\section{MATERIALS AND METHODS}

\section{Preparation of Oligonucleotides}

DNAs (including FAM/biotin-labeled aptamers) were synthesized by Shanghai Sangon Biological Engineering Technology \& Services (Shanghai, China). The BC15-31s in the animal experiment and the LNA- and $2^{\prime}$-O-MOE-modified BC15-31s were synthesized using Qingke-DNAchem-12 synthesizer according to the literature procedure using standard phosphoramidite chemistry (Huang et al., 2013, 2015; Ma et al., 2017). The standard iodine solution was replaced by phenylacetyl disulfide solution (5 $\mathrm{g}$ in $2 \mathrm{ml}$ pyridine) in the oxidation step to complete the PS modification. Since phenylacetyl disulfide has no stereoselectivity, the synthesized oligonucleotides are racemates. Cleavage was performed in concentrated ammonia/40\% methylamine water solution $(1 / 1$ $\mathrm{vol} / \mathrm{vol}$ ) at $65^{\circ} \mathrm{C}$ for $90 \mathrm{~min}$. The $\mathrm{BC} 15-31$ variants were purified by $\mathrm{C} 18$ reverse high-performance liquid chromatography (XBridge $^{\mathrm{TM}}$ OST C18, $2.5 \mu \mathrm{m}, 10 \mathrm{~mm} \times 50 \mathrm{~mm}$ ) using a linear gradient of 5 to $40 \%$ eluent $\mathrm{A}$ for $25 \mathrm{~min}$. $\mathrm{CH}_{3} \mathrm{CN}$ was used as eluent $A$, and a solution of $0.05 \mathrm{M}\left(\mathrm{Et}_{3} \mathrm{~N}\right)_{2} \mathrm{CO}_{3}$ in water was used as eluent $\mathrm{B}$; the column temperature is $25^{\circ} \mathrm{C}$, and the flow rate is $1.5 \mathrm{ml} / \mathrm{min}$. Then, the isolated $5^{\prime}$-DMTprotected oligonucleotides were treated with $80 \%$ acetic acid at room temperature for $10 \mathrm{~min}$. After neutralized with $\mathrm{Et}_{3} \mathrm{~N}$, the oligonucleotide solutions were desalted by Sephadex G25 column, respectively. The oligonucleotide compositions were confirmed by MALDI-TOF-MS spectrometry (Supplementary Figures 2-42).

\section{Cell Culture and Nanocomplex Preparation}

A375 and SK-MEL-2 cells were grown in Dulbecco's modified Eagle's medium (M\&C, Beijing, China), BcPAP, SW-480, MCF7, MCF-7/ADR, A549, A549/Taxol, and HepG-2 cells were grown in Roswell Park Memorial Institute (RPMI)-1640 medium (M\&C), supplemented with $10 \%$ fetal bovine serum (Gibco, Grand Island, United States) at $37^{\circ} \mathrm{C}$ and $5 \% \mathrm{CO}_{2}$ in a humidified incubator. The DNCA and CLD lipids were dissolved in ethanol, respectively, as stock solutions $(1 \mathrm{mM})$. The aptamers were dissolved in $\mathrm{H}_{2} \mathrm{O}$ at a concentration of $0.05 \mathrm{nmol} / \mu \mathrm{l}$ as stock solutions. Then, DNCA, CLD, and aptamers were diluted to appropriate concentrations with GenOpti (M\&C) and sonicated for $20 \mathrm{~min}$ under $70^{\circ} \mathrm{C}$. The nanocomplexes were obtained and diluted to a certain concentration for usage.

\section{Cellular Antiproliferation Assay}

The CCK-8 assay was used to evaluate the cytotoxicity and the antiproliferative activity of nanocomplexes (concentration of aptamers: $25 \mathrm{nM})$. A549/Taxol $\left(4 \times 10^{3}\right.$ per well), A375, SKMEL-2, MCF-7, MCF-7/ADR, A549 cells $\left(5 \times 10^{3}\right.$ per well), BcPAP, SW-480, and HepG-2 cells $\left(1 \times 10^{4}\right.$ per well $)$ were seeded 
TABLE 1 | The sequences of phosphothioated aptamer BC15-31s.

\begin{tabular}{|c|c|c|}
\hline No. & Name & Sequence $\left(5^{\prime}-3^{\prime}\right)$ \\
\hline 1 & WW-1 & $\begin{array}{l}\text { T"G*T*GGCGAGGTAGGTGGGGTGT } \\
\text { GTGTGTATC }\end{array}$ \\
\hline 2 & WW-2 & TGTG*G*C*GAGGTAGGTGGGGTGTGTGTGTATC \\
\hline 3 & WW-3 & TGTGGC* ${ }^{*} A^{*}$ GGTAGGTGGGGTGTGTGTGTATC \\
\hline 4 & WW-4 & TGTGGCGAG* ${ }^{*}{ }^{*} T^{*}$ AGGTGGGGTGTGTGTGTATC \\
\hline 5 & WW-5 & TGTGGCGAGGTA* G* G*TGGGGTGTGTGTGTATC \\
\hline 6 & WW-6 & TGTGGCGAGGTAGGT"G*G*GGTGTGTGTGTATC \\
\hline 7 & WW-7 & TGTGGCGAGGTAGGTGGG* G*T*GTGTGTGTATC \\
\hline 8 & WW-8 & TGTGGCGAGGTAGGTGGGGTG* ${ }^{*}{ }^{*}{ }^{*}$ TGTGTATC \\
\hline 9 & WW-9 & TGTGGCGAGGTAGGTGGGGTGTGT"G*T"GTATC \\
\hline 10 & $W W-10$ & TGTGGCGAGGTAGGTGGGGTGTGTGTG ${ }^{*} T^{*} A^{*}$ TC \\
\hline 11 & WW-11 & TGTGGCGAGGTAGGTGGGGTGTGTGTGT*A*T*C \\
\hline 12 & WW-12 & T*G*T*G*GCGAGGTAGGTGGGGTGTGTGTGTATC \\
\hline 13 & $W W-13$ & TGTGGCGAGG*T*A*G*GTGGGGTGTGTGTGTATC \\
\hline 14 & WW-14 & TGTGGCGAGGTAGGTG* ${ }^{*}{ }^{*}{ }^{*}$ G $^{*}$ TGTGTGTGTATC \\
\hline 15 & WW-15 & TGTGGCGAGGTAGGTGGGGTGT*G*T*G*TGTATC \\
\hline 16 & WW-16 & TGTGGCGAGGTAGGTGGGGTGTGTGTG ${ }^{*} T^{*} A^{*} T^{*} \mathrm{C}$ \\
\hline 17 & BC15-31 & TGTGGCGAGGTAGGTGGGGTGTGTGTGTATC \\
\hline 18 & WW-18 & T*G*T*GGCGAGGTAGGTGGGGTGTGTGTGT"A*T"C \\
\hline 19 & WW-19 & T"G $^{*} T^{*}$ GGCGAGGTA* ${ }^{*}$ G $^{*}$ TGGGGTGTGTGTGT"A* ${ }^{*} T^{*} \mathrm{C}$ \\
\hline 20 & $W W-20$ & $\begin{array}{l}T^{*} G^{*} T^{*} G^{*} G^{*} C^{*} G^{*} A^{*} G^{*} G^{*} T^{*} A^{*} G^{*} G^{*} T^{*} G^{*} G^{*} G \\
{ }^{*} G^{*} T^{*} G^{*} T^{*} G^{*} T^{*} G^{*} T^{*} G^{*} T^{*} A^{*} T^{*} C\end{array}$ \\
\hline 21 & NC & TGGTGCGGTGTGGGTCGAGTGGTATGGTATG \\
\hline 22 & $W W-23$ & T"G $^{*} T^{*}$ GGCGAG*G*T*AGGTGGGGTGTGTGTGT"A*T*C \\
\hline 23 & WW-24 & $\begin{array}{l}T^{*} G^{*} T^{*} G G C G A G^{*} G^{*} T^{*} A^{*} G^{*} G^{*} T G \\
\text { GGGTGTGTGTA } A^{*} T^{*} C\end{array}$ \\
\hline 24 & 24-L1 & $\begin{array}{l}T_{L N A}{ }^{*} G^{*} T^{*} G G C G A G^{*} G^{*} T^{*} A^{*} G^{*} G^{*} T G G G G \\
\text { TGTGTGTG }{ }^{*} A^{*} T^{*} C\end{array}$ \\
\hline 25 & 24-L2 & $\begin{array}{l}T^{*} G^{*} T_{L N A}{ }^{*} G G C G A G^{*} G^{*} T^{*} A^{*} G^{*} G^{*} T G G G G T \\
\text { GTGTGTGT } A^{*} T^{*} C\end{array}$ \\
\hline 26 & 24-L3 & $\begin{array}{l}T^{*} G^{*} T^{*} G G C G A G^{*} G^{*} T_{L N A}{ }^{*} A^{*} G^{*} G^{*} T G G G G \\
\text { TGTGTGTGTA*A"T }\end{array}$ \\
\hline 27 & 24-L4 & $\begin{array}{l}T^{*} G^{*} T^{*} G G C G A G * G^{*} T^{*} A^{*} G^{*} G^{*} T \text { LNA GGGGT } \\
\text { GTGTGTGTA*A*T"C }\end{array}$ \\
\hline 28 & 24-L5 & $\begin{array}{l}T^{*} G^{*} T^{*} G G C G A G G^{*} G^{*} T^{*} A^{*} G^{*} G^{*} T G G G G T_{L N A} G T \\
\text { GTGTGTA*T"C }\end{array}$ \\
\hline 29 & 24-L6 & 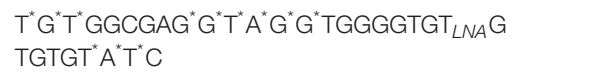 \\
\hline 30 & 24-L7 & $\begin{array}{l}T^{*} G^{*} T^{*} G G C G A G^{*} G^{*} T^{*} A^{*} G^{*} G^{*} T G G G G T G T G T_{L N A} G \\
\text { TGT }^{*} A^{*} T^{*} C\end{array}$ \\
\hline 31 & 24-L8 & $\begin{array}{l}T^{*} G^{*} T^{*} G G C G A G^{*} G^{*} T^{*} A^{*} G^{*} G^{*} T G G G G \\
\text { TGTGTGT } T_{L N A} G T^{*} A^{*} T^{*} C\end{array}$ \\
\hline 32 & 24-L9 & $\begin{array}{l}T^{*} G^{*} T^{*} G G C G A G^{*} G^{*} T^{*} A^{*} G^{*} G^{*} T G G G G \\
\text { TGTGTGTGT } L N A A^{*} A^{*} T^{*} C\end{array}$ \\
\hline 33 & 24-L10 & $\begin{array}{l}T^{*} G^{*} T^{*} G G C G A G^{*} G^{*} T^{*} A^{*} G^{*} G^{*} T G G G \\
\text { GTGTGTGTGT"A } T_{\text {LNA }}{ }^{*} C\end{array}$ \\
\hline 34 & 24-M1 & $\begin{array}{l}T_{M O E}{ }^{*} G_{M O E}{ }^{*} T^{*} \mathrm{GGG}_{M O E} \mathrm{C}_{M O E} G A G^{*} \mathrm{G}^{*} T^{*} A^{*} \mathrm{G}^{*} \mathrm{G}^{*} \text { TGGGGT } \\
\text { GTGTGTGTA*T"C }\end{array}$ \\
\hline 35 & 24-M2 & $\begin{array}{l}T^{*} G^{*} T_{M O E}{ }^{*} G_{M O E} G C G M O E A_{M O E} G^{*} G^{*} T^{*} A^{*} G^{*} G^{*} T G G G G T \\
\text { GTGTGTGT'A } A^{*} T^{*} C\end{array}$ \\
\hline 36 & 24-M3 & $\begin{array}{l}T^{*} G^{*} T^{*} G G C G A G_{M O E}{ }^{*} G_{M O E}{ }^{*} T^{*} A^{*} G_{M O E}{ }^{*} G_{M O E}{ }^{*} \text { TGGGGTG } \\
\text { TGTGTG } A^{*} T^{*} C\end{array}$ \\
\hline 37 & 24-M4 & $\begin{array}{l}T^{*} G^{*} T^{*} G G C G A G^{*} G^{*} T_{M O E}{ }^{*} A_{M O E}{ }^{*} G^{*} G^{*} T_{M O E} G_{M O E} G \\
\text { GGTTGTGTA }{ }^{*} T^{*} C\end{array}$ \\
\hline 38 & 24-M5 & $\begin{array}{l}T^{*} G^{*} T^{*} G G C G A G^{*} G^{*} T^{*} A^{*} G^{*} G^{*} T_{G G} G_{M O E} G_{M O E} G_{T} G_{M O E} T \\
\text { MOEGTGTGT"A*T"C }\end{array}$ \\
\hline
\end{tabular}

(Continued)
TABLE 1 | Continued

\begin{tabular}{|c|c|c|}
\hline No. & Name & Sequence $\left(5^{\prime}-3^{\prime}\right)$ \\
\hline 39 & 24-M6 & $\begin{array}{l}T^{*} G^{*} T^{*} G G C G A G^{*} G^{*} T^{*} A^{*} G^{*} G^{*} T G G G G_{M O E} T_{M O E} G T \\
G_{M O E} T_{M O E} G T G T^{*} A^{*} T^{*} C\end{array}$ \\
\hline 40 & 24-M7 & $\begin{array}{l}T^{*} G^{*} T^{*} G G C G A G{ }^{*} G^{*} T^{*} A^{*} G^{*} G^{*} T G G G G T G T G T M_{M O E} T \\
\text { MOE GT"A MOE } T^{*} \text { MOE C }\end{array}$ \\
\hline 41 & 24-M8 & $\begin{array}{l}T^{*} G^{*} T^{*} G G C G A G^{*} G^{*} T^{*} A^{*} G^{*} G^{*} \text { TGGGGTGTGTGTGMOE } \\
{ }^{M O E} A^{*} T^{*} C\end{array}$ \\
\hline
\end{tabular}

" ") represents phosphorothioate modification site; "T LNA" represents LNA-T

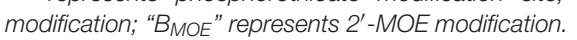

into a 96-well plate and incubated with the nanocomplexes for $48 \mathrm{~h}$. The group incubated with the nanocomplex of the negative control sequence (NC: 5'-TGG TGC GGT GTG GGT CGA GTG GTA TGG TATG-3') was used as the blank. According to standard, $10 \mu \mathrm{l}$ CCK-8 solution (Dojindo Laboratories, Beijing, China) was added to a 96-well plate. The absorbance at $450 \mathrm{~nm}$ was measured by a microplate reader (Molecular Devices, San Francisco, CA, United States). The relative cell viability was calculated by the equation:

$$
\left[\left(R_{A}-R_{E}\right) /\left(R_{B}-R_{E}\right)\right] \times 100 \%
$$

( $R_{A}, R_{B}$, and $R_{E}$ are defined as the absorbance of experimental samples, untreated samples, and blank controls, respectively).

\section{Flow Cytometry Assay}

A375 cells $\left(4 \times 10^{4}\right.$ per well) were cultured in 24 -well plates and proliferated for 16-24 h. FAM-labeled aptamers were used to prepare the nanocomplexes (FAM-labeled aptamers: $25 \mathrm{nM}$ ). The nanocomplexes and FAM-labeled aptamers were exposed to cells and incubated for $4 \mathrm{~h}$, respectively. Subsequently, the cells were harvested and centrifuged at $1,000 \times g$ for $3 \mathrm{~min}$. The precipitates were washed by precooled phosphate-buffered saline (PBS) and filtered for a homogeneous distribution in the solution. The cellular uptake was observed on a CytoFLEX (Beckman Coulter, South Kraemer Boulevard, Brea, CA, United States) immediately.

\section{Confocal Microscopy Assay}

A375 cells $\left(1.4 \times 10^{5}\right.$ per well $)$ were cultured at confocal observation dishes and proliferated for 16-24 h. FAM-labeled aptamers were used to prepare the nanocomplexes. The nanocomplexes and FAM-labeled aptamers were exposed to cells and incubated for $4 \mathrm{~h}$, respectively. Subsequently, the culture medium was removed, followed by washing twice with PBS. Cells were stained with Hoechst 33342 (Solarbio, Beijing, China) for $20 \mathrm{~min}$ and washed twice with PBS and then observed under an A1Rsi confocal microscope (Nikon Instruments Inc., Tokyo, Japan). Confocal images were obtained using NIS-Elements software (Nikon Instruments Inc.).

\section{Sizes, Zeta Potentials, and Transmission Electron Microscopy Morphology Assay}

The aptamer lipoplexes (aptamer: $10 \mu \mathrm{M}$ ) and free lipids were diluted in PBS, following filtration by a $0.22-\mu \mathrm{m}$ filter. Then, particle sizes and $\zeta$ potentials were measured at $25^{\circ} \mathrm{C}$ and 


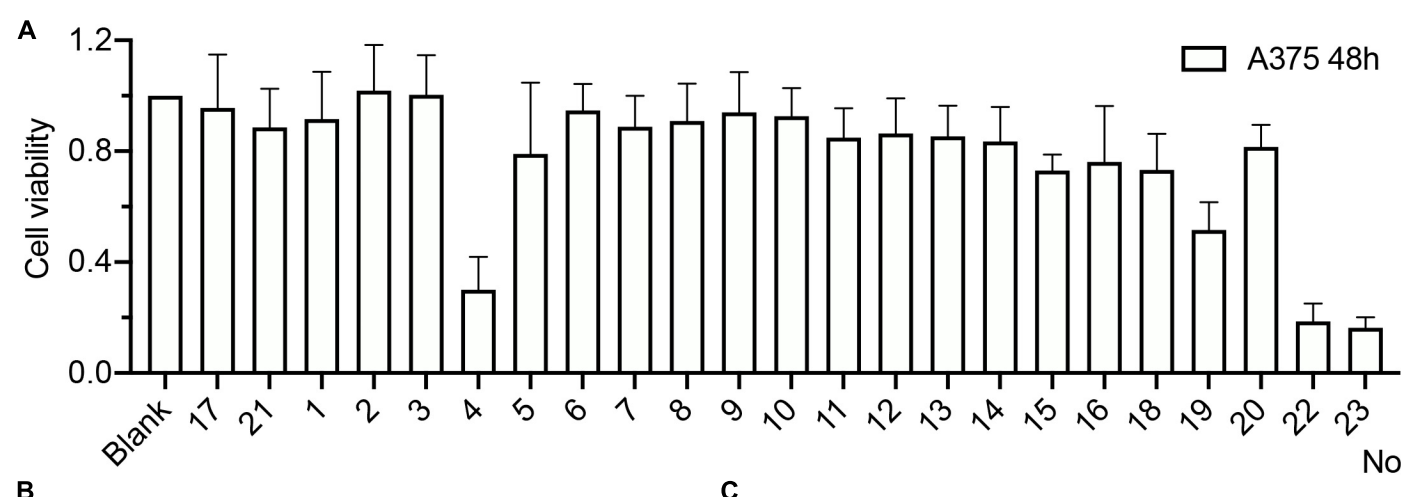

B

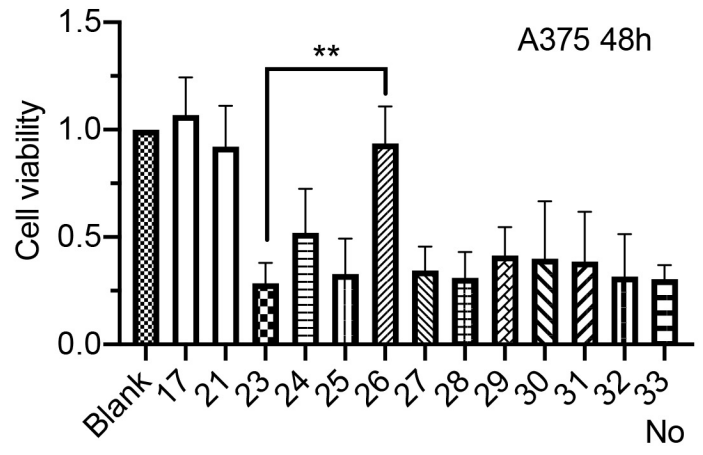

C

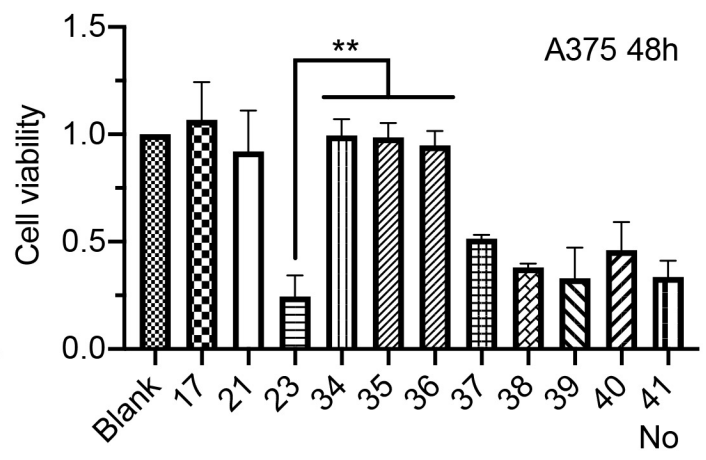

FIGURE 1 | Antiproliferation activity of chemical modified BC15-31s (DNCA/CLD/nt: 4:1:1.25 nM; 48 h) in A375 cell line. (A) Proliferation inhibitory effect of aptamer with PS modification. (B) Proliferation inhibitory effect of aptamer with LNA modification. (C) Proliferation inhibitory effect of aptamer with $2^{\prime}$-O-MOE modification. Numbers "1," "2," "3," etc. correspond to the serial number in Table 1. Experimented with CCK-8 reagent, ${ }^{* \star} P<0.01$.

analyzed using dynamic light scattering (DLS) (Malvern Zetasizer Nano ZS, Worcestershire, United Kingdom). The morphology of lipoplexes was examined using transmission electron microscopy (TEM, JEM-1400Plus, 120 kV, Jeol, Tokyo, Japan). The lipoplexes were negatively stained with $1 \%$ uranyl acetate.

\section{Apoptosis Assay}

Cellular apoptosis was quantified using an Annexin V-APC/PI Apoptosis Detection Kit (KeyGen Biotech, Nanjing, China). After treatment with nanocomplexes of $\mathrm{BC} 15-31$ and $\mathrm{WW}-24$, the A375 cells seeded into six-well plates were harvested after $24 \mathrm{~h}$ of incubation. Subsequently, the cells were washed with cold PBS twice and resuspended in $500 \mu \mathrm{l}$ of $1 \times$ binding buffer. Next, $5 \mu \mathrm{l}$ Annexin V-APC and $5 \mu \mathrm{l}$ PI were added to each sample, and the cells were incubated at room temperature for $20 \mathrm{~min}$ in the dark. The stained samples were then determined by CytoFLEX (Beckman Coulter).

\section{Surface Plasmon Resonance Experiments}

Surface plasmon resonance (SPR) experiments were performed on Biacore T100 (GE Healthcare, Pittsburgh, PA, United States) to know the binding kinetics of aptamers and hnRNP A1. The commercially purchased gold substrates CM5 (Thermo Fisher, Waltham, MA, United States) were used as the SPR sensor chips.
The reagents N-hydroxysuccinimide (NHS) and N-ethyl-N'-(3diethylaminopropyl) carbodiimide hydrochloride (EDC) were used to activate the sensor chips, and recombined protein hnRNP A1 (ProSpec-Tany TechnoGene Ltd., Ness-Ziona, Israel) was linked on the sensor chips. Then, the unreacted active carboxyl intermediate was blocked with ethanolamine. The running buffer for immobilization and sample analysis was $10 \mathrm{mM}$ phosphatebuffered saline (138 mM NaCl, $2.7 \mathrm{mM} \mathrm{KCl,} 10 \mathrm{mM} \mathrm{Na}_{2} \mathrm{HPO}_{4}$, $1.76 \mathrm{mM} \mathrm{KH}_{2} \mathrm{PO}_{4}, 0.05 \%$ Tween-20), $\mathrm{pH} \mathrm{7.4}$, at $25^{\circ} \mathrm{C}$. Every aptamer solution was sequentially injected over the sensor surface for $3 \mathrm{~min}$ at $30 \mu \mathrm{l} / \mathrm{min}$ and $5 \mathrm{~min}$ dissociation time. For each sample, six aptamer concentrations were injected by serially diluting samples from 0.39 to $50 \mathrm{nM}$ and a blank sample only containing running buffer for referencing. The unbound aptamer was removed by treatment with $4.0 \mathrm{M} \mathrm{NaCl}$ aqueous, and the chip was primed before use. The raw data were processed and analyzed to determine the binding constant for each oligonucleotide. In order to correct refractive index changes and instrument noise, the response data from the control surface were subtracted from the responses obtained from the reaction surface using BIA evaluation 4.1 software.

\section{Serum Stability of PS-Modified Aptamers}

Aptamer (140 pmol) was diluted to $56 \mu \mathrm{l}$ with PBS. The $56-\mu \mathrm{l}$ sample was divided equally into seven tubes, and $2 \mu \mathrm{l}$ fetal bovine serum was added into six tubes, respectively. One tube was added with $2 \mu \mathrm{l}$ PBS as the blank group. The solutions were incubated 

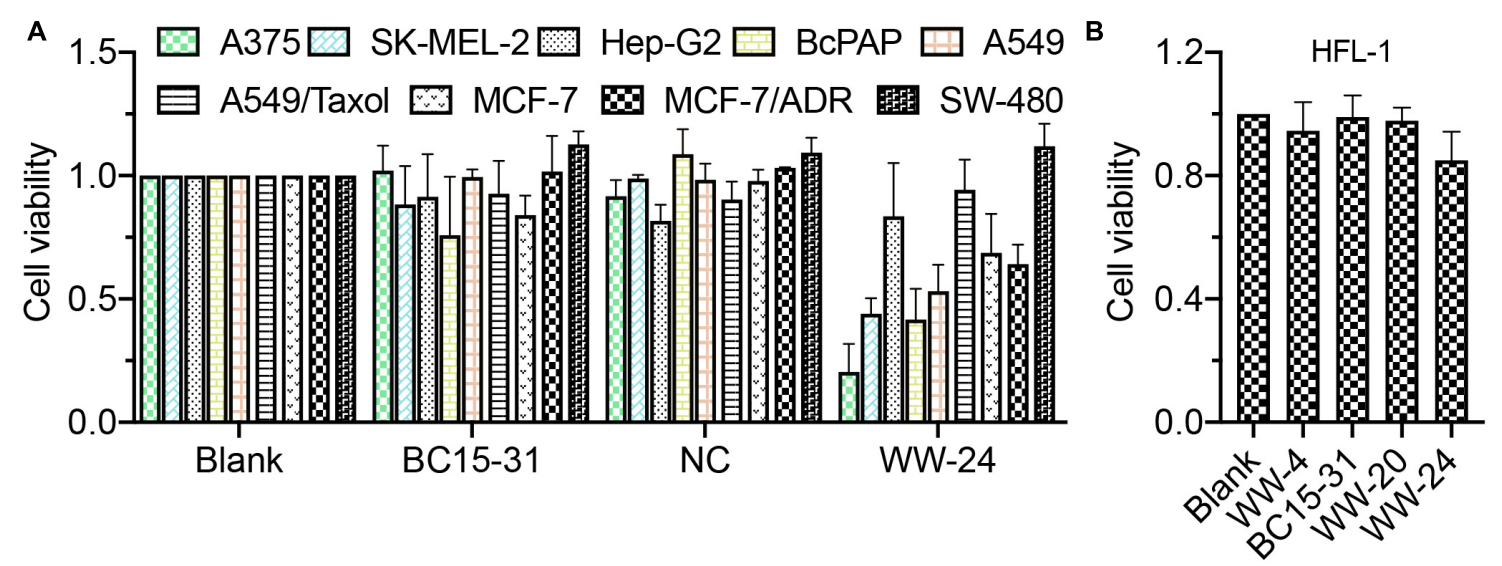

FIGURE 2 | The cell viability of various cell lines with lipid/aptamer nanocomplexes (DNCA/CLD/nt: 1:1:1, aptamers: 25 nM, 48 h). (A) The proliferation inhibition of aptamers on tumor cell lines. (B) The proliferation inhibition of aptamers on human normal cell line. Experimented with CCK-8 reagent.

A

\begin{tabular}{cccc}
\hline Name & Zeta potential $(\mathbf{m V})$ & Size $(\mathbf{d} . \mathbf{n m})$ & PDI \\
\hline Mix & $30.5 \pm 1.6$ & $156 \pm 21.4$ & $0.44 \pm 0.07$ \\
BC15-31/Mix & $34.0 \pm 4.2$ & $155 \pm 25.4$ & $0.17 \pm 0.01$ \\
WW-24/Mix & $25.8 \pm 7.4$ & $141 \pm 10.2$ & $0.16 \pm 0.03$ \\
\hline
\end{tabular}

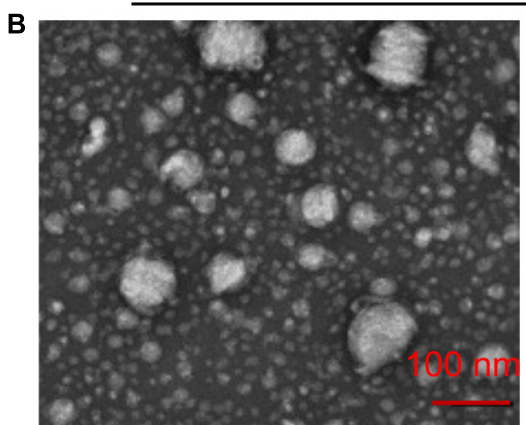

Mix

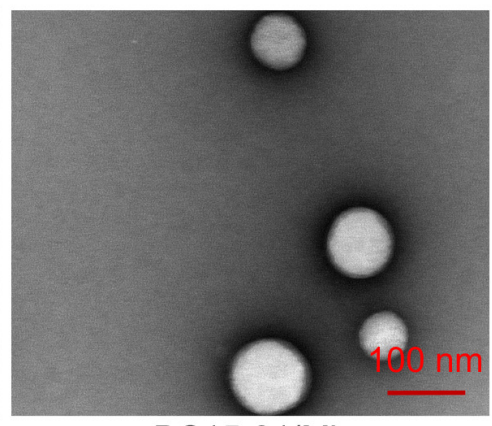

BC15-31/Mix

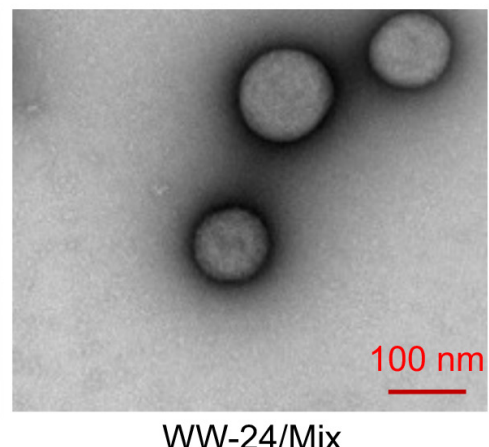

WW-24/Mix

FIGURE 3 | Characterization of DNCA/CLD/aptamer liposomes. (A) The size, zeta potential, and PDI of the nanocomplexes. (B) TEM result of DNCA/CLD/aptamer nanoparticles (DNCA/CLD/nt: 1/1/1; aptamers: $10 \mu \mathrm{M}$ ).

at $37^{\circ} \mathrm{C}$ for $0,0.5,1,2,4$, and $8 \mathrm{~h}$. Each tube was removed and inactivated at $95^{\circ} \mathrm{C}$ for $5 \mathrm{~min}$, and then placed in a $-80^{\circ} \mathrm{C}$ refrigerator for detection. All samples were resolved with $20 \%$ polyacrylamide denaturing (7 M urea) gel electrophoresis and visualized by staining with SYBR-Gold and quantified by using a ChemiDoc XRS highly sensitive chemiluminescence gel imaging analyzer (Bio-Rad, Hercules, CA, United States).

\section{Target Fishing Experiment}

Biolayer interferometry (BLI) was used to investigate the target of WW-24. WW-24 was biotinylated at the $5^{\prime}$-terminal (Sangon, China). A375 cell lysate was prepared by the nuclear protein extraction kit (Solarbio, Beijing, China). Biotin-WW-24 was diluted to $5 \mu \mathrm{g} / \mu \mathrm{l}$ and loaded to six SSA sensors for $420 \mathrm{~s}$, and the sensors were infiltrated in PBS for $60 \mathrm{~s}$ and then put into
A375 cell lysate for fishing. We can see a strong association only in the experiment group and nearly no association in the blank group. After fishing for $300 \mathrm{~s}$, the sensors were inserted into $0.1 \%$ formic acid solution for the wash. The experiment was repeated 100 times, and the formic acid solution of the experiment and blank groups was gathered up, respectively, for MS analysis.

\section{Animal Experiments of Antitumor Efficiency}

The committee approved all animal experiments for animal research of Peking University (no. LA2017194). In addition, all of the operations on animals conformed to the National Institutes of Health Guide for the Care and Use of Laboratory Animals (NIH Publications no. 8023, revised 1978). Specific 

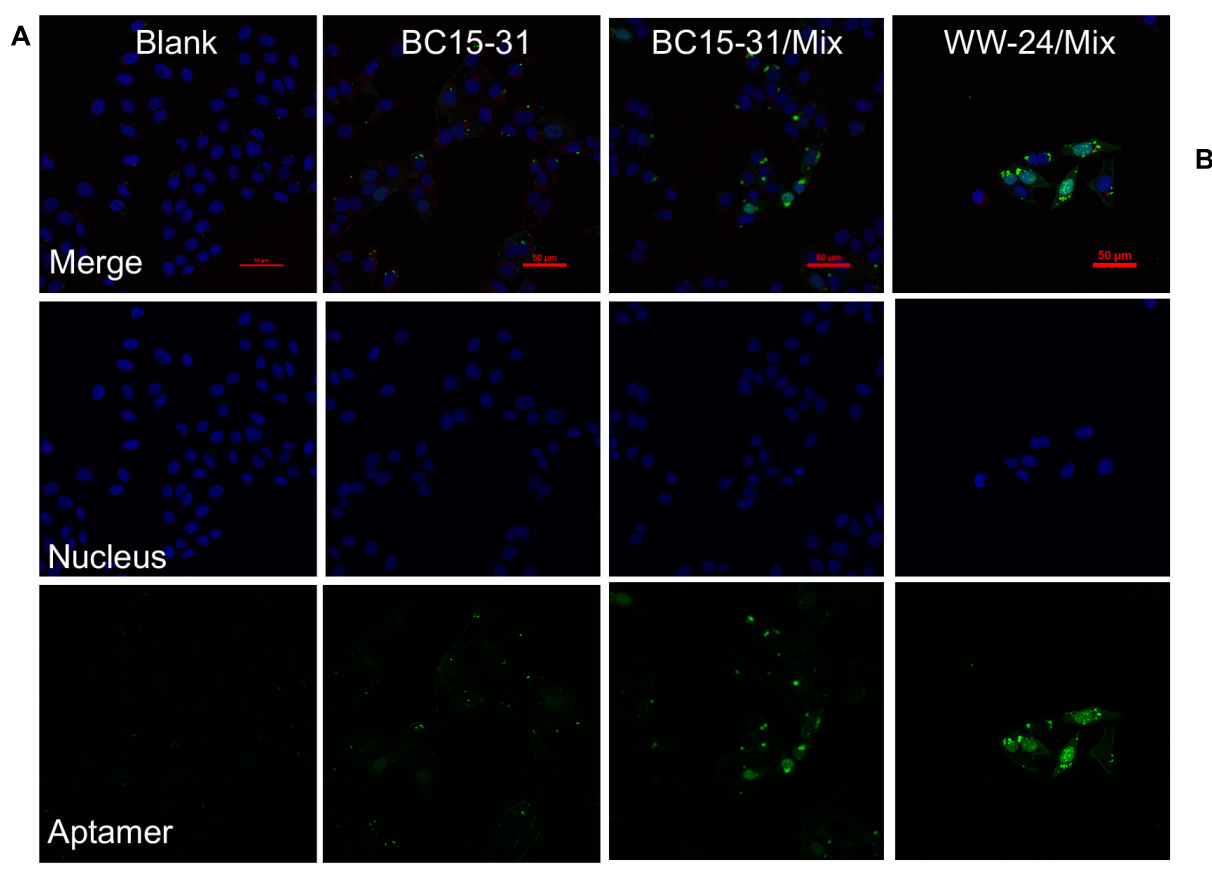

B

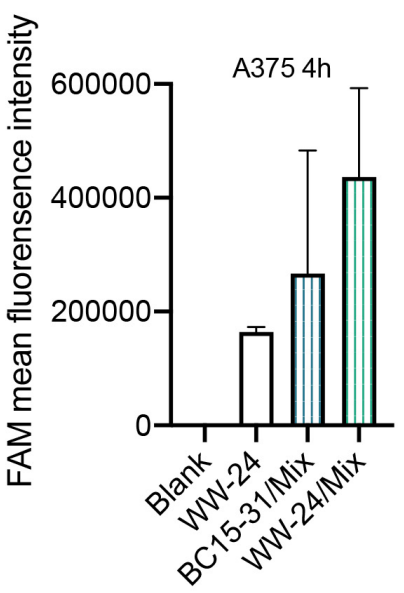

FIGURE 4 | Liposomes improve transmembrane ability of aptamers. (A) Confocal analysis of lipid/aptamer nanocomplex permeation (FAM-aptamers, $25 \mathrm{nM}$ ). The cells were observed at $6 \mathrm{~h}$ after incubation with blank, FAM-BC15-31, FAM-BC15-31/Mix, and FAM-WW-24/Mix nanocomplexes, in which the aptamer and nucleus were visible by green and blue colors. (B) Cellular uptake of lipid/aptamer nanocomplex permeation (FAM-aptamers, $25 \mathrm{nM}, n=3$ ) at $4 \mathrm{~h}$. All data in this study are average values, and error bars represent standard deviations.

pathogen-free (SPF)-grade female BALB/c nude mice (3-4 weeks) were obtained from Wantonglihua (China) and kept at the Department of Laboratory Animal Science, Peking University Health Science Center.

A375 cells $\left(1.6 \times 10^{6}\right)$ were subcutaneously inoculated into the mice. When the tumor volumes reached $50-80 \mathrm{~mm}^{3}$ in size, the mice were randomly divided into four groups, which were treated with GenOpti (free solvent, blank), NC/Mix nanocomplex, BC15-31/Mix nanocomplex, and WW-24/Mix nanocomplex $(2.0 \mathrm{mg} / \mathrm{kg})$ by peritumoral injection, respectively. The mice $(n=5)$ received the treatment at days $1,3,5,7$, and 9 . The tumor volumes $\left(\mathrm{mm}^{3}\right)$ were calculated by equations:

$$
\text { Volume }=\text { length } \times \text { width } 2 \times 0.5
$$

$$
\text { Re }=\text { Volume/Volume } 1
$$

[Re, Volume, and Volume 1 were defined as the relative tumor volume, practical tumor volume, and original (day 1) tumor volume, respectively.]

\section{Biodistribution Assay in vivo}

Female BALB/c nude mice (3-4 weeks) bore xenografted A375 cells $\left(1.6 \times 10^{6}\right)$ subcutaneously under the right arm. When the tumor volume reached about $1,200 \mathrm{~mm}^{3}$, different formulations consisting of Cy7-labeled aptamers, DNCA, CLD, and PEGDSPE were injected by the peritumoral an aptamer concentration of $1.5 \mathrm{mg} / \mathrm{kg}$. The images were taken at $1,2,4,8,12$, and
$24 \mathrm{~h}$ after injection utilizing the IVIS Spectrum in vivo imaging system (PerkinElmer, Shanghai, China). The measurements were performed at $745 \mathrm{~nm}$ excitation wavelength and $800 \mathrm{~nm}$ emission wavelength. The mice were sacrificed at $24 \mathrm{~h}$ time points, and the tumors and organs were then isolated for the ex vivo distribution assay.

\section{Statistical Analysis}

All data in this study are average values, and error bars represent standard deviations. Two groups of independent data were analyzed by $t$-test, and one-way ANOVA analyzed multiple groups of independent data. Significance of $P$-value: n.s. means $P>0.05$; $^{*}$ means $P<0.05$; ** means $P<0.01$; *** means $P<0.001$. All statistical analyses were performed using GraphPad Prism 8.0 software.

\section{RESULTS}

\section{Design of Liposome and Screening of Modification Sites}

The PS modification of DNA can influence the properties of oligonucleotide in various ways, such as serum stability and protein-binding capacity ( $\mathrm{Hu}$ et al., 2015). An excess of PS modification sites will enhance the protein-binding ability, but it may decrease the special binding with its target protein simultaneously, which indicated that the PS backbone should only be modified at necessary sites; this is the key to improving 


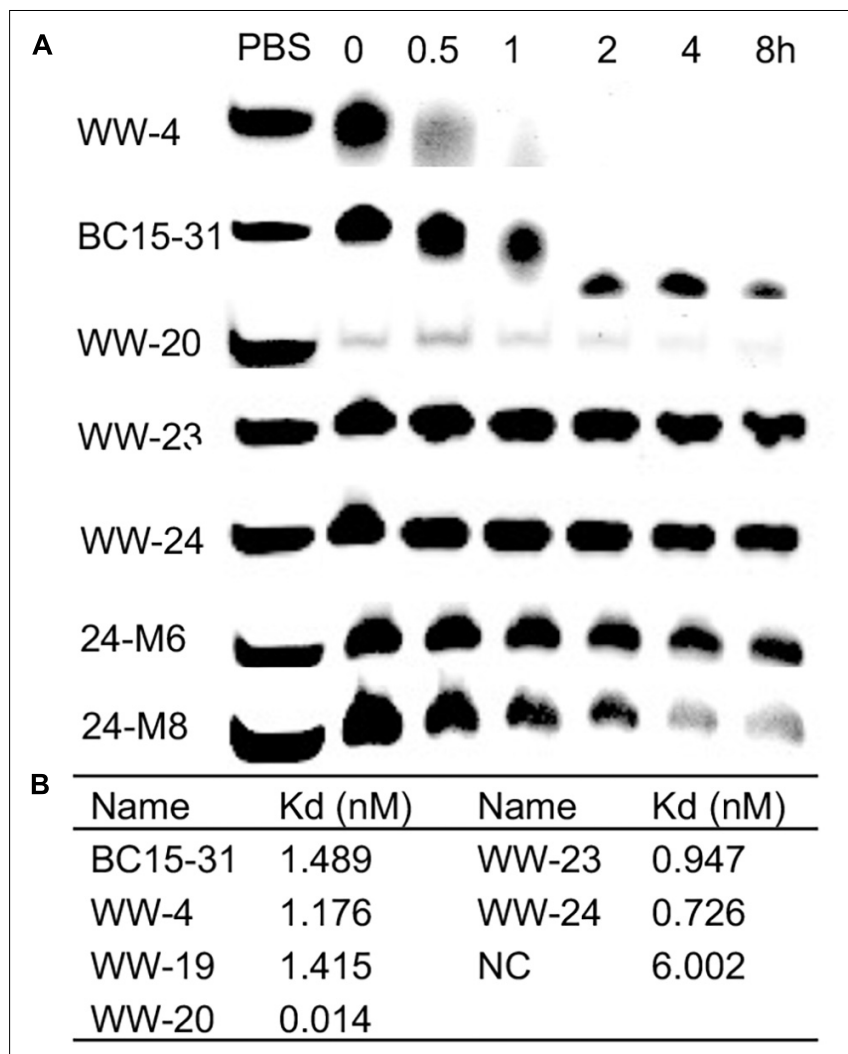

FIGURE 5 | (A) Serum stability of representative PS-modified BC15-31s (20\% FBS; 20 pmol aptamer). (B) Binding affinity of PS-modified BC15-31s to hnRNP A1.

its specific target binding affinity. LNA imposes a conformational restriction to adopt an N-type sugar puckering (Koshkin et al., 1998). The LNA modification strategy could improve the properties of the oligonucleotides, thereby increasing their potential for therapeutic purposes. LNA modification could improve the biological stability and target affinity of aptamers (Schmidt et al., 2004; Lebars et al., 2007). 2'-O-MOE is usually used as the second-generation modification strategy of antisense nucleic acid to improve its pharmacokinetic properties, reduce the immunostimulatory side effects caused by PS modification, and improve its affinity (Manoharan, 1999).

Three consecutive positions of PS-modified BC15-31s (WW-1 $\sim \mathrm{WW}-20$ ) were synthesized (Table 1), while mixed DNCA/CLD(Mix) was used to encapsulate the BC15-31s at an empirical molecular ratio (DNCA:CLD:aptamer $=124: 31: 1$ ). We treated the A375 cell at a concentration of $25 \mathrm{nM}$ to investigate the antiproliferation. After $48 \mathrm{~h}, \mathrm{WW}-4$ and WW-19 (Table 1) showed good antiproliferation activity (Figure 1A). Based on that of WW-4 and WW-19, two sequences WW23 and WW-24 were designed and synthesized (Table 1), which showed excellent antitumor activity. We discerned that the concentration of $25 \mathrm{nM}$ is too low for an unmodified aptamer BC15-31 to exert an ideal antitumor activity. The antiproliferation activity of the NC group clearly revealed that the cytotoxicity of mixed lipids is very low at this concentration.
Ten LNA-T-modified sequence, 24-L1 24-L10, as well as eight 2'-O-MOE-modified sequences, 24-M1 24-M8 (Table 1), were further designed and synthesized. However, no individual showed significant biological improvements compared with WW-24 (Figures 1B,C). Further optimization of the preparation DNCA:CLD:aptamer = 31:31:1 could give identical biological effect with WW-23 and WW-24 (Supplementary Figure 1).

As hnRNPs were overexpressed in most tumor cells, MCF-7, M/A, A549, A/T, HepG2, SK-MEL-2, BcPAP, SW480, and A375 cell lines were treated with WW-24/Mix at $25 \mathrm{nM}$ (Figure 2A), which showed strong antiproliferation effect on the A375 cell line; moderate on the SK-MEL-2, BcPAP, and A549 cell lines; and almost no activity on the others. On human normal cell line HFL-1, the following preparations (Figure 2B) were found to be almost non-toxic.

\section{Characterization of DNCA/CLD/aptamer Liposome}

Dynamic Light Scattering (DLS) was used to investigate the particle size and zeta potentials of BC15-31/Mix and WW24/Mix. The results (Figure 3A) showed that were around 150 $\mathrm{nM}$ and $34 \pm 4.2$ and $25.8 \pm 7.4 \mathrm{mV}$, respectively. A transmission electron microscope (TEM) was then used to verify the size of liposomes again. Without aptamers, the surface of liposomes will shrink (Figure 3B). The WW-24/Mix nanoparticles can form uniform spherical nanoparticles with smooth edges. In summary, the BC15-31s/Mix can form spherical structures with sizes between 130 and $\sim 180 \mathrm{~nm}$, and the surfaces were positively charged.

\section{Transmembrane Assay of DNCA/CLD Enhances Aptamers}

Confocal analysis was performed to investigate the subcellular distribution of BC15-31/Mix and WW-24/Mix (Figure 4A). The results showed that the nanoparticles could be delivered into cells with high efficiency. Without a DNCA/CLD encapsulation, the BC15-31 aptamer had almost no fluorescence at $6 \mathrm{~h}$. Compared with BC15-31/Mix, WW-24/Mix was significantly enriched in cells after $6 \mathrm{~h}$; this may be attributed to the better stability by PS modification. Both BC15-31 and WW-24 preferred to go into the nucleus. Flow cytometry experiments showed that, compared with the non-encapsulated BC15-31 (Figure 4B), BC15-31s/Mix had improved ability to deliver the aptamers into cells. Specifically, WW-24 was more stable in cells compared with BC15-31.

\section{Phosphorothioate Modification Promotes BC15-31's Stability and Target Affinity}

In 1967, Eckstein (1967) (De Clercq et al., 1969) first reported the strategy of PS modification to enhance the ribonucleases (RNase) resistance of RNA. Some of the BC15-31s with good anti-proliferation activities were selected, which were incubated in $20 \%$ fetal bovine serum. PAGE results showed that, compared with BC15-31, the serum stability of $3^{\prime}$-terminal modified 


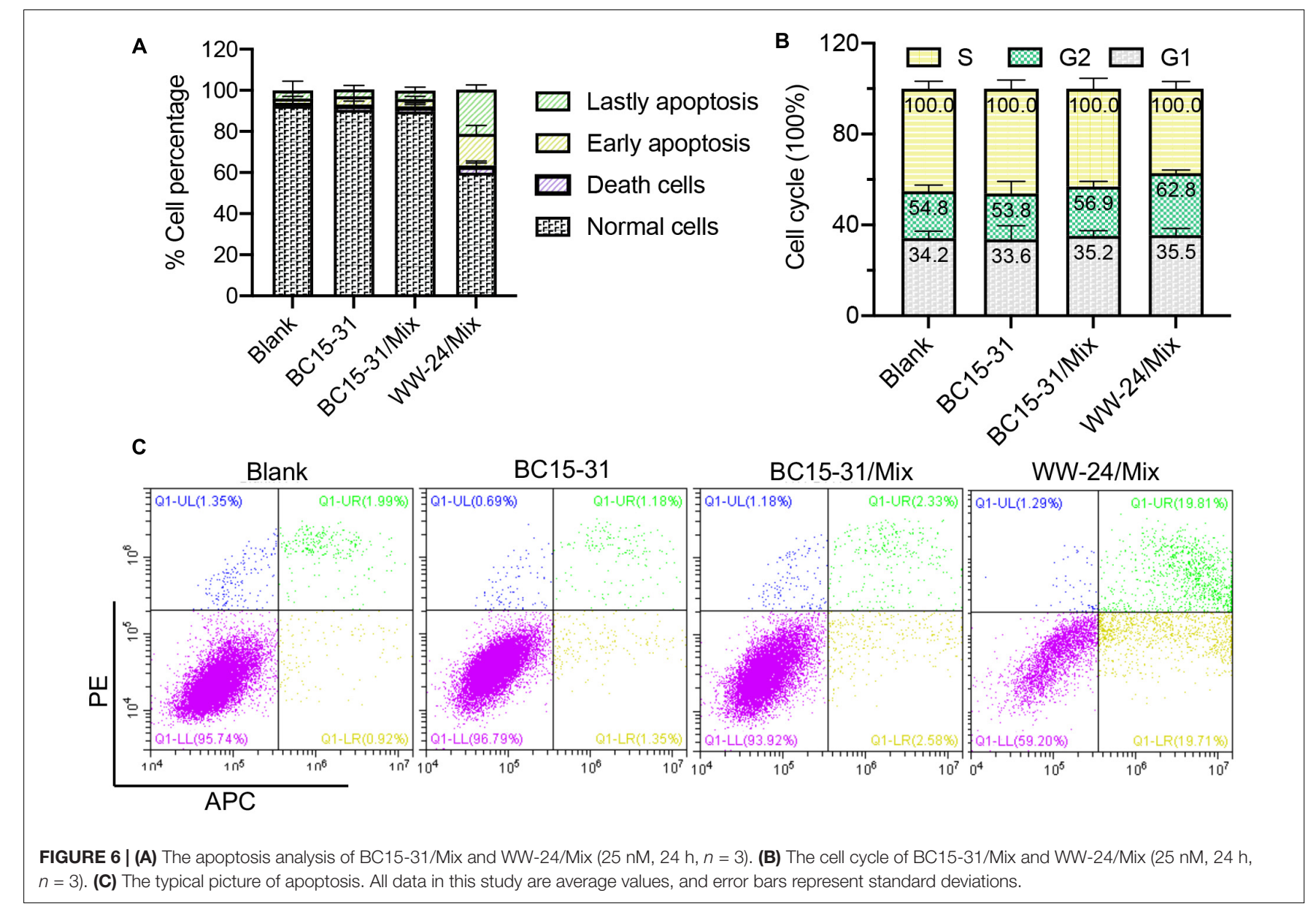

TABLE 2 | Target protein of aptamer WW-24.

\begin{tabular}{llll}
\hline Protein & PBS & NC & WW-24 \\
\cline { 2 - 4 } & PSMs & PSMs & PSMs \\
\hline Heterogeneous nuclear ribonucleoprotein A1 & 25 & 111 & 281 \\
Heterogeneous nuclear ribonucleoproteins A2/B1 & 24 & 63 & 229 \\
Heterogeneous nuclear ribonucleoprotein U & - & 79 & 81 \\
Desmoplakin & 42 & 28 & 85 \\
Heterogeneous nuclear ribonucleoprotein D0 & 8 & 55 & 39
\end{tabular}

aptamers (including WW-19, WW-20, WW-23, WW-24) were dramatically improved (Figure 5A).

SPR experiments were performed to investigate the target affinity of BC15-31s. We immobilized the protein hnRNP A1 instead of aptamers on the chip, using the amino coupling strategy. BC15-31s that showed good antitumor activities were selected to investigate the target affinity. The results showed that the target affinities of all modified BC15-31s were improved compared with BC15-31 (Figure 5B). All site PS-modified sequence WW-20 showed the strongest binding ability (14 pM), which was very hard to regenerate. It might be the reason why it does not show ideal antitumor activity due to nonspecial binding.

\section{Mix-WW-24 Promotes the Process of Apoptosis}

Different from cell necrosis, apoptosis is an active process involving the activation, expression, and regulation of a series of genes (Reed, 2000). The apoptosis effects of BC1531/Mix and WW-24/Mix on A375 cell were investigated. The results (Figure 6A) showed that, compared with BC1531 , WW-24 can promote early and late apoptosis ( $235 \%)$, while $7 \%$ of the cell cycle is blocked in the G2/M phase (Figure 6B). The typical picture of apoptosis were shown here (Figure 6C).

\section{Target Fishing Experiment}

BLI was used to "fish" the target of WW-24 from the A375 cell. Nuclear protein was extracted, since the aptamers were mainly distributed in the nucleus. We coupled biotin to the $5^{\prime}$-terminal of WW-24 and loaded it to the SA sensors and then put the sensors into the nuclear protein extraction solutions. Table 2 showed the results of our experiment. Here, a strong combination was observed compared with the blank group. After that, the sensors were pulled out and inserted into formic acid solutions to desorb the binding proteins. Thus, the binding proteins will be enriched in formic acid solutions, and then MS analysis was performed. 
A

$1 \mathrm{~h}$

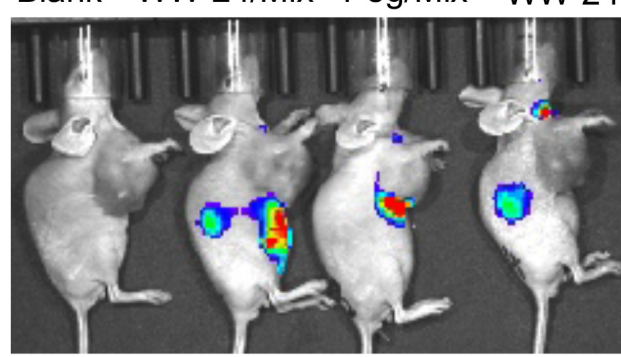

$2 \mathrm{~h}$

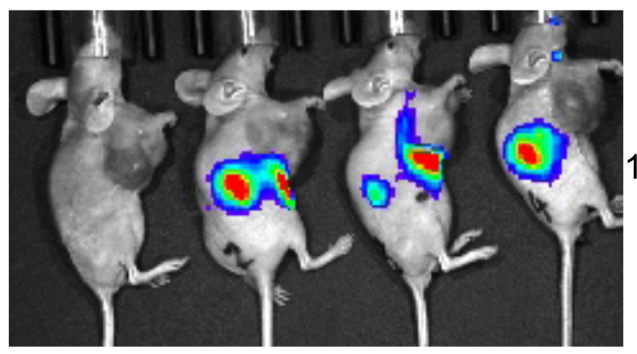

B

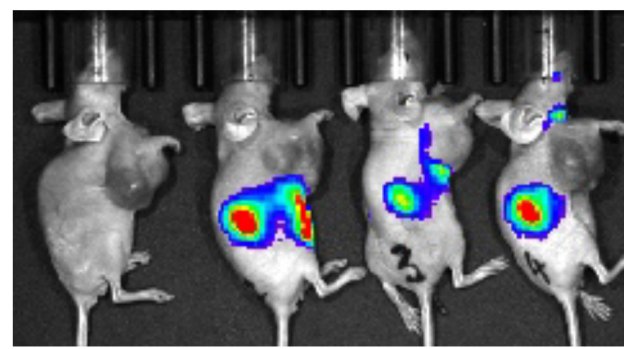

Blank WW-24/Mix Peg/Mix WW-24
$8 h$
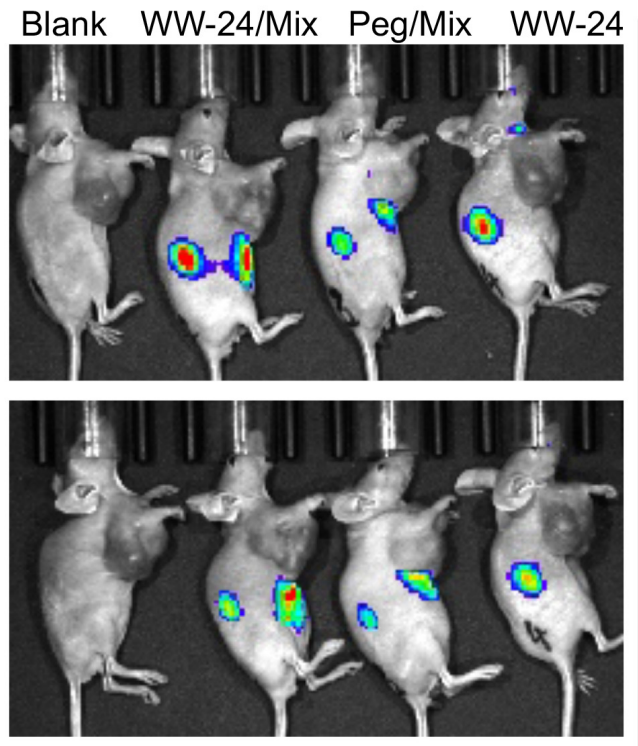

$24 \mathrm{~h}$

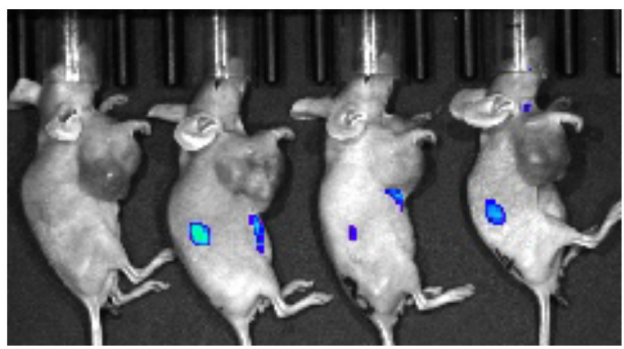

Blank WW-24/Mix Peg/Mix WW-24
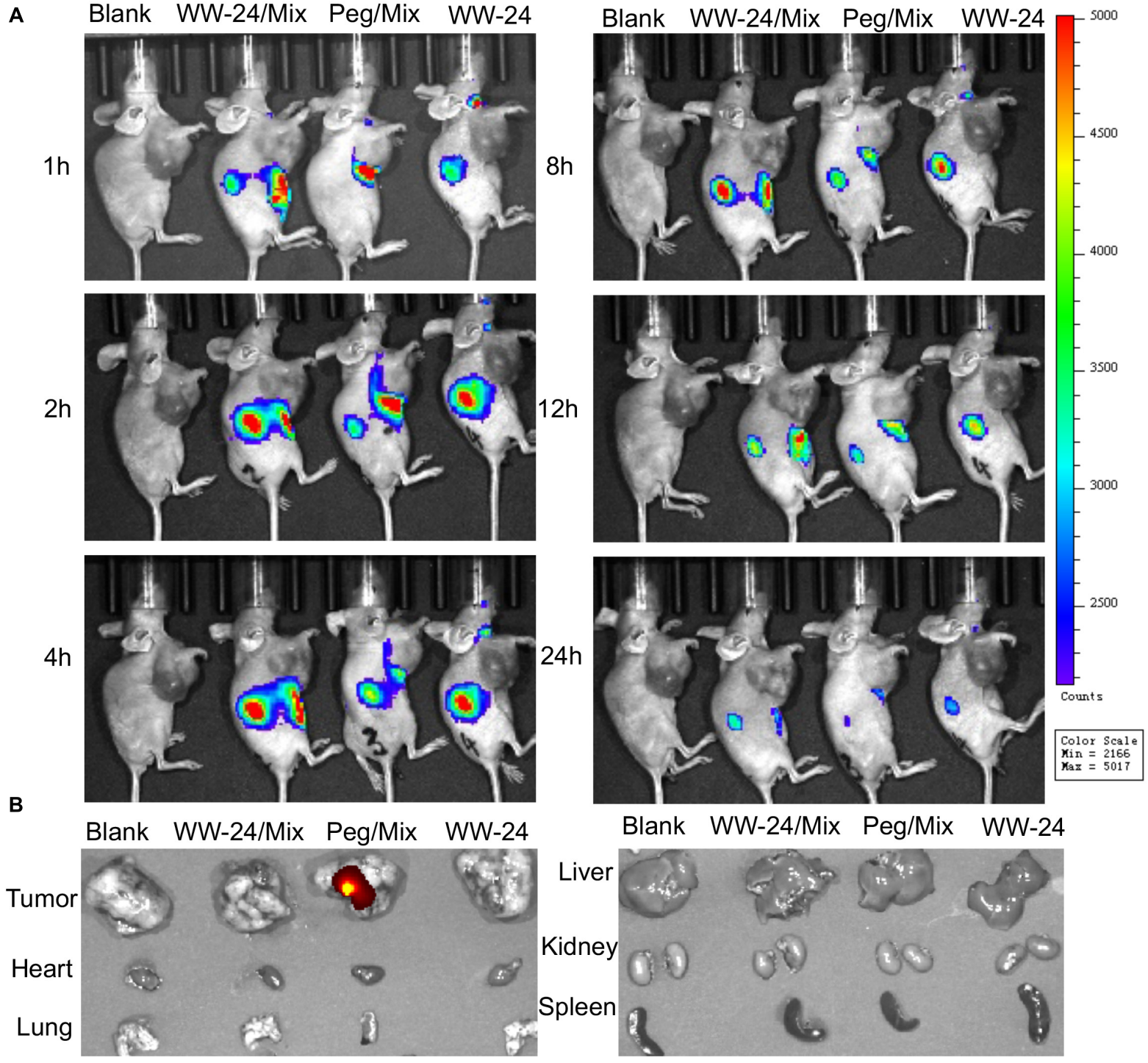

Liver

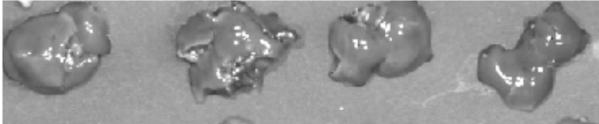

Kidney
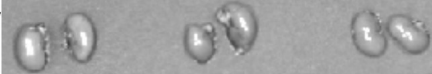

Spleen
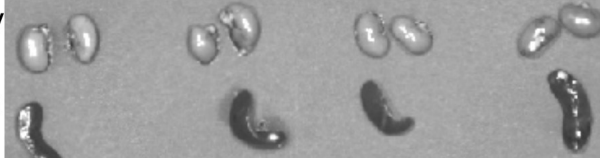

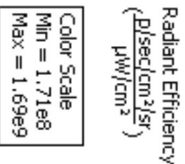

is

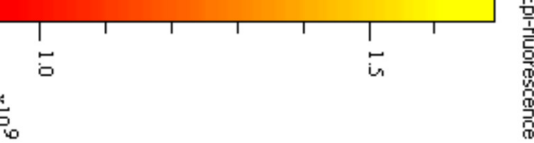

FIGURE 7 | Distribution of nanoparticles of WW-24/Mix with 1\% PEG-2000 in vivo by peritumoral injection. The biodistribution of Cy7-labeled WW-24 was observed by an IVIS spectrum in vivo imaging system at the excitation wavelength of $745 \mathrm{~nm}$ and emission wavelength of $800 \mathrm{~nm}$. (A) Typical in vivo imaging at $1,2,4,8,12$, and $24 \mathrm{~h}$ (the black arrows show the tumor tissues). (B) Imaging of organs and tumors at $48 \mathrm{~h}$ excised from BALB/c nude mice ex vivo.

The results showed that the abundance of hnRNPs in the experimental group was significantly higher than that of the blank group. We concluded that the most different proteins, hnRNP $\mathrm{A} 2 / \mathrm{B} 1$ and hnRNPA1, were the key target proteins related to the proliferation activity of WW-24. The specificity of the aptamers is not as good as that of antibodies because the hydrophobic interaction and the electrostatic interaction made outstanding contributions to the stable 3D structure formation of antibody, and this did not play an essential role in the structure formation of the aptamers. In addition, the aptamers are smaller than antibodies, which means that the aptamers may bind to similar substructures of different proteins and that hnRNP A1 has the same domain structure as hnRNP A2/B1 (Dreyfuss et al., 2002).

\section{Biodistribution}

In order to extend the metabolism time of the aptamers in vivo and enhance the stability of the nanoparticles, $1 \%$ PEG was introduced into the liposome system (Peg/Mix). After the peritumoral injection, we determined that there was almost no difference in tissue distribution; however, when the mice were 


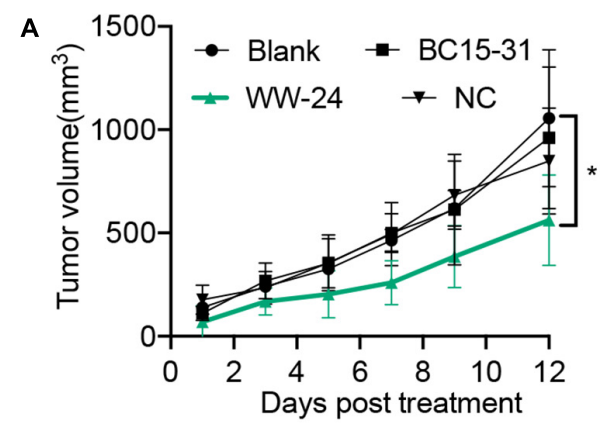

B

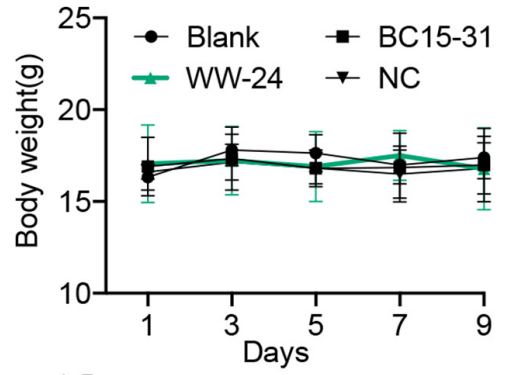

C

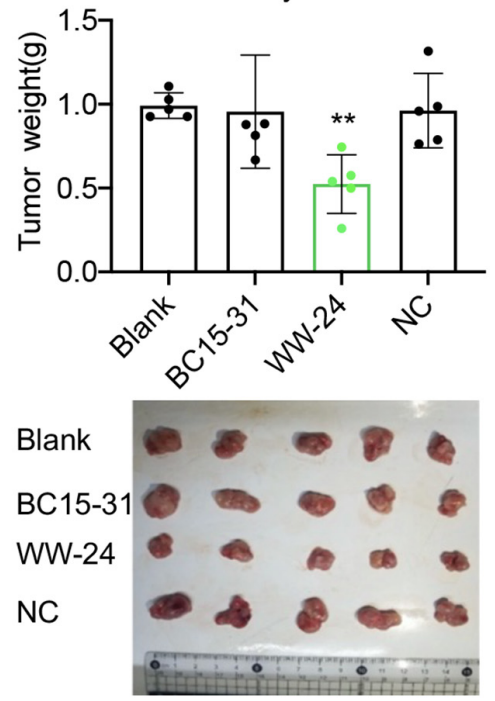

FIGURE 8 | Antitumor efficiency and biosafety of WW-24/Mix nanocomplexes in vivo. A375 cells were inoculated into BALB/c mice. When the tumors had reached $50-80 \mathrm{~mm}^{3}$, the mice were treated with GenOpti (blank) or aptamers/Mix by peritumoral injection ( $2 \mathrm{mg} / \mathrm{kg}$ ). (A) Tumor volumes were measured and calculated $\left(n=5,{ }^{*} P<0.05\right)$. (B) Body weights of mice treated with nanocomplexes $(n=5)$. (C) The comparison of each group tumor weight and the bar chart. Statistical analysis was carried out utilizing multiple $t$-test one per row, and statistical significance was corrected using the Holm-Sidak method for post hoc analysis (A/B). ${ }^{\star \star} P<0.01$. (D) The picture of each group tumor. All data in this study are average values, and error bars represent standard deviations.

dissected after $48 \mathrm{~h}$, the PEG group showed a better tumor accumulation (Figure 7).

\section{Antitumor Efficiency and Toxicity of WW-24/liposome in vivo}

Subcutaneous A375 tumors in the BALB/c mice model were established to evaluate the antitumor efficiency and safety in vivo.
Twenty mice were divided into four groups $(n=5)$; these groups were then treated with GenOpti (blank), NC/Mix, BC15$31 / \mathrm{Mix}$, and WW-24/Mix by peritumoral injection (2 $\mathrm{mg} / \mathrm{kg}$ ), respectively. The results showed that the tumor of the WW24/Mix group was relatively smaller than those of the other groups (Figure 8A) after 12 days $(P<0.05)$. There was no difference in the body weights between the four groups, and all 20 mice survived for 12 days (Figure 8B). After 12 days, the tumors of all the mice were taken out and weighed. We found that the tumors of the mice in the WW-24/Mix group were significantly smaller $(P<0.01)$ than those of the other three groups (Figures $\mathbf{8 C}, \mathbf{D})$.

\section{DISCUSSION}

Similar to monoclonal antibodies, aptamers can specifically recognize and bind targets. Therefore, aptamers have many applications in diagnosis and therapy, such as biosensors, targeted therapy, and target inhibitors. In terms of disease treatment, aptamers can activate target receptors to affect their biological functions or serve as carriers for drug delivery to target cells or tissues. Exogenous nucleic acid drugs need to overcome multiple obstacles in order to enter the body to work (Singh et al., 2018).

Because of the limitations encountered in the delivery of nucleic acid drugs in vivo, various measures have been applied to solve the above problems. The more important ones are the chemical modification (Adachi and Nakamura, 2019) of nucleotides and the application of delivery systems (Baillet et al., 2018). To improve the stability of nucleic acid molecules and reduce their immunogenicity, the development of delivery system technology has made it possible to prevent nucleic acid drugs from being degraded by nucleases while improving the efficiency of their entry into cells.

The serum stability and the target affinity of aptamer BC15-31 were improved by partial PS, LNA-T, and $2^{\prime}-O-$ MOE modification. A sequence WW-24 showed excellent antiproliferation activity on the A375 cell line at low concentrations $(25 \mathrm{~nm}, \sim 85 \%)$. In addition, the cytidinyl lipid DNCA and cationic lipid CLD were mixed at the optimal ratio to the single nucleotide(nt) (DNCA:CLD:nt $=1: 1: 1$ ) as a low-toxic vector that can deliver WW-24 and reduce the dose to $25 \mathrm{nM}$. WW-24 can promote the apoptotic transformation of cancer cells in the late stage and has an obvious influence on the cell cycle. The fishing experiment showed that WW24 can specifically bind to hnRNP A1 and hnRNP A2/B1. An intratumoral injection was used to act on the periphery of the tumor directly. It has not been confirmed that the EPR effect enables the drug to target tumor cells for a long time. Also, the pathway and mechanism of aptamer entry are not yet clear, and the binding mode of the target is also a direction that needs to be explored in the future. Although flow cytometry experiments have confirmed the phenomenon of apoptosis, the specific mechanism is still unknown. Overall, as an anticancer agent, WW-24/Mix has excellent application prospects. This provided a new idea for drug development based on aptamers. 


\section{DATA AVAILABILITY STATEMENT}

The original contributions presented in the study are included in the article/Supplementary Material, further inquiries can be directed to the corresponding author.

\section{ETHICS STATEMENT}

The animal study was reviewed and approved by The Committee for Animal Research of Peking University (no. LA2017194).

\section{AUTHOR CONTRIBUTIONS}

ZY conceived the project. SW and JW designed the experiment. JW performed the main experiments, analyzed the data, and reviewed the literatures and edited the manuscript. SW was responsible for most of the synthesis of the phosphothioated aptamers, performed the experiments, and wrote the original draft. XL was responsible for synthesizing the 2'-O-MOEmodified aptamers. QZ, JY, YM, and ZG provided experimental

\section{REFERENCES}

Adachi, T., and Nakamura, Y. (2019). Aptamers: a review of their chemical properties and modifications for therapeutic application. Molecules 24, 42294243. doi: 10.3390/molecules24234229

Baillet, J., Desvergnes, V., Hamoud, A., Latxague, L., and Barthelemy, P. (2018). Lipid and nucleic acid chemistries: combining the best of both worlds to construct advanced biomaterials. Adv. Mater. 30:1705078. doi: 10.1002/adma. 201705078

Burd, C. G., and Dreyfuss, G. (1994). RNA binding specificity of hnRNP A1: significance of hnRNP A1 high-affinity binding sites in pre-mRNA splicing. Embo J. 13, 1197-1204.

Chovelon, B., Fiore, E., Faure, P., Peyrin, E., and Ravelet, C. (2018). Mirror-image aptamer kissing complex for arginine-vasopressin sensing. Anal. Chim. Acta 1001, 143-150. doi: 10.1016/j.aca.2017.11.043

Cui, S., Wang, Y., Gong, Y., Lin, X., Zhao, Y., Zhi, D., et al. (2018). Correlation of the cytotoxic effects of cationic lipids with their headgroups. Toxicol. Res. 7 , 473-479. doi: 10.1039/c8tx00005k

De Clercq, E., Eckstein, E., and Merigan, T. C. (1969). Interferon induction increased through chemical modification of a synthetic polyribonucleotide. Science 165, 1137-1139. doi: 10.1126/science.165.3898.1137

Ding, J., Hayashi, M. K., Zhang, Y., Manche, L., Krainer, A. R., Xu, R. M., et al. (1999). Crystal structure of the two-RRM domain of hnRNP A1 (UP1) complexed with single-stranded telomeric DNA. Gene Dev. 13, 1102-1115. doi: 10.1101/gad.13.9.1102

Dowling, P., Pollard, D., Larkin, A., Henry, M., Meleady, P., Gately, K., et al. (2015). Abnormal levels of heterogeneous nuclear ribonucleoprotein A2B1 (hnRNPA2B1) in tumour tissue and blood samples from patients diagnosed with lung cancer. Mol. Biosyst. 11, 743-752. doi: 10.1039/c4mb00384e

Dreyfuss, G., Kim, V. N., and Kataoka, N. (2002). Messenger-RNA-binding proteins and the messages they carry. Nat. Rev. Mol. Cell Bio. 3, 195-205. doi: $10.1038 / \mathrm{nrm} 760$

Eckstein, F. (1967). A dinucleoside phosphorothioate. Tetrahedron Lett. 8, 1157 1160. doi: 10.1016/S0040-4039(00)90656-7

Filion, M. C., and Phillips, N. C. (1997). Toxicity and immunomodulatory activity of liposomal vectors formulated with cationic lipids toward immune effector cells. Biochim. Biophys. Acta 1329, 345-356. doi: 10.1016/s0005-2736(97) 00126-0

He, Y., Brown, M. A., Rothnagel, J. A., Saunders, N. A., and Smith, R. (2005). Roles of heterogeneous nuclear ribonucleoproteins A and B in cell proliferation. J. Cell Sci. 118, 3173-3183. doi: 10.1242/jcs.02448 support. All authors contributed to the article and approved the submitted version.

\section{FUNDING}

This work was supported by the Ministry of Science and Technology of China (Grant No. 2017ZX09303013) and the National Natural Science Foundation of China (Grant No. 21778006).

\section{SUPPLEMENTARY MATERIAL}

The Supplementary Material for this article can be found online at: https://www.frontiersin.org/articles/10.3389/fcell.2021. 660233/full\#supplementary-material

Supplementary Figure 1 The antiproliferation activity of WW-24 encapsulated in different ratios of DNCA/CLD upon A375 (25 nM).

Supplementary Figure 2-42 | The ESI-MS of 41 aptamers.

Supplementary Figure 43-49 | The gel pattern of Figure 5A.

Hermann, T., and Patel, D. J. (2000). Biochemistry-Adaptive recognition by nucleic acid aptamers. Science 287, 820-825. doi: 10.1126/science.287.5454.820

Hoerter, J. A. H., and Walter, N. G. (2007). Chemical modification resolves the asymmetry of siRNA strand degradation in human blood serum. RNA 13, 1887-1893. doi: 10.1261/rna.602307

Hu, Y., Duan, J., Cao, B., Zhang, L., Lu, X., Wang, F., et al. (2015). Selection of a novel DNA thioaptamer against HER2 structure. Clin. Transl. Oncol. 17, 647-656. doi: 10.1007/s12094-015-1292-0

Huang, Y., Chen, Z., Chen, Y., Zhang, H., Zhang, Y., Zhao, Y., et al. (2013). Effects of conformational alteration induced by $\mathrm{D}$-/L-isonucleoside incorporation in siRNA on their stability in serum and silencing activity. Bioconjug. Chem. 24, 951-959. doi: 10.1021/bc300642u

Huang, Y., Tian, M., Zhang, Y., Sheng, G., Chen, Z., Ma, Y., et al. (2015). DIsonucleotide (isoNA) incorporation around cleavage site of passenger strand promotes the vibration of Ago2-PAZ domain and enhances in vitro potency of siRNA. Org. Biomol. Chem. 13, 10825-10833. doi: 10.1039/c5ob01119a

Knudsen, K. B., Northeved, H., Kumar, P. E., Permin, A., Gjetting, T., Andresen, T. L., et al. (2015). In vivo toxicity of cationic micelles and liposomes. Nanomedicine 11, 467-477. doi: 10.1016/j.nano.2014.08.004

Koshkin, A. A., Nielsen, P., Meldgaard, M., Rajwanshi, V. K., Singh, S. K., Wengel, J., et al. (1998). LNA (locked nucleic acid): an RNA mimic forming exceedingly stable LNA : LNA duplexes. J. Am. Chem. Soc. 120, 13252-13253. doi: 10.1021/ ja9822862

Ku, T. H., Zhang, T. T., Luo, H., Yen, T. M., Chen, P. W., Han, Y., et al. (2015). Nucleic acid aptamers: an emerging tool for biotechnology and biomedical Sensing. Sensors 15, 16281-16313. doi: 10.3390/s150716281

Lebars, I., Richard, T., Di Primo, C., and Toulme, J. J. (2007). LNA derivatives of a kissing aptamer targeted to the trans-activating responsive RNA element of HIV-1. Blood Cells Mol. Dis. 38, 204-209. doi: 10.1016/j.bcmd.2006. 11.008

Li, L., Yang, X. T., Li, K., Zhang, G., Ma, Y., Cai, B., et al. (2018). D-/L-Isothymidine incorporation in the core sequence of aptamer BC15 enhanced its binding affinity to the hnRNP A1 protein. Org. Biomol. Chem. 16, 7488-7497. doi: $10.1039 /$ c8ob01454j

Li, S., Wang, W., Ding, H. M., Xu, H., Zhao, Q., Li, J., et al. (2012). Aptamer BC15 against heterogeneous nuclear ribonucleoprotein A1 has potential value in diagnosis and therapy of hepatocarcinoma. Nucleic Acid Ther. 22, 391-398. doi: $10.1089 /$ nat.2012.0363

Li, S., Xu, H., Ding, H., Huang, Y., Cao, X., Yang, G., et al. (2009). Identification of an aptamer targeting hnRNP A1 by tissue slide-based SELEX. J. Pathol. 218, 327-336. doi: 10.1002/path.2543 
Ma, X. F., Sun, J., Qiu, C., Wu, Y. F., Zheng, Y., et al. (2016). The role of disulfidebridge on the activities of $\mathrm{H}$-shape gemini-like cationic lipid based siRNA delivery. J Control Release 235, 99-111. doi: 10.1016/j.jconrel.2016.05.051

Ma, Y., Liu, S., Wang, Y., Zhao, Y., Huang, Y., Zhong, L., et al. (2017). Isonucleotide incorporation into middle and terminal siRNA duplexes exhibits high gene silencing efficacy and nuclease resistance. Org. Biomol. Chem. 15, 5161-5170. doi: $10.1039 / \mathrm{c} 7 \mathrm{ob} 01065 \mathrm{f}$

Ma, Y., Zhao, W., Li, Y., Pan, Y., Wang, S., Zhu, Y., et al. (2019). Structural optimization and additional targets identification of antisense oligonucleotide G3139 encapsulated in a neutral cytidinyl-lipid combined with a cationic lipid in vitro and in vivo. Biomaterials 197, 182-193. doi: 10.1016/j.biomaterials. 2018.12.033

Ma, Y., Zhu, Y., Wang, C., Pan, D., Liu, S., Yang, M., et al. (2018). Annealing novel nucleobase-lipids with oligonucleotides or plasmid DNA based on H-bonding or pi-pi interaction: assemblies and transfections. Biomaterials 178, 147-157. doi: 10.1016/j.biomaterials.2018.06.012

Manoharan, M. (1999). 2 '-Carbohydrate modifications in antisense oligonucleotide therapy: importance of conformation, configuration and conjugation. Biochim. Biophys. Acta 1489, 117-130. doi: 10.1016/S01674781(99)00138-4

Mascini, M., Palchetti, I., and Tombelli, S. (2012). Nucleic acid and peptide aptamers: fundamentals and bioanalytical aspects. Angew. Chem. Int. Ed Engl. 51, 1316-1332. doi: 10.1002/anie.201006630

Neriec, N., and Percipalle, P. (2018). Sorting mRNA molecules for cytoplasmic transport and localization. Front. Genet. 9:510. doi: 10.3389/fgene.2018.00510

Park, S. J., Jung, Y. H., Kim, Y. G., and Park, H. J. (2008). Identification of novel ligands for the RNA pseudoknot that regulate-1 ribosomal frameshifting. Bioorg. Med. Chem. 16, 4676-4684. doi: 10.1016/j.bmc.2008.02.025

Pino, I., Pio, R., Toledo, G., Zabalegui, N., Vicent, S., Rey, N., et al. (2003). Altered patterns of expression of members of the heterogeneous nuclear ribonucleoprotein (hnRNP) family in lung cancer. Lung Cancer 41, 131-143. doi: 10.1016/s0169-5002(03)00193-4

Reed, J. C. (2000). Mechanisms of apoptosis. Am. J. Pathol. 157, 1415-1430. doi: 10.1016/S0002-9440(10)64779-7

Sahay, G., Alakhova, D. Y., and Kabanov, A. V. (2010). Endocytosis of nanomedicines. J. Control Release 145, 182-195. doi: 10.1016/j.jconrel.2010.0 1.036

Schmidt, K. S., Borkowski, S., Kurreck, J., Stephens, A. W., Bald, R., Hecht, M., et al. (2004). Application of locked nucleic acids to improve aptamer in vivo stability and targeting function. Nucleic Acids Res. 32, 5757-5765. doi: 10.1093/ nar/gkh862

Singh, A., Trivedi, P., and Jain, N. K. (2018). Advances in siRNA delivery in cancer therapy. Artif. Cells Nanomed. Biotechnol. 46, 274-283. doi: 10.1080/21691401. 2017.1307210
Tok, J. B., Cho, J., and Rando, R. R. (2000). RNA aptamers that specifically bind to a $16 \mathrm{~S}$ ribosomal RNA decoding region construct. Nucleic Acids Res. 28, 2902-2910. doi: 10.1093/nar/28.15.2902

Ushigome, M., Ubagai, T., Fukuda, H., Tsuchiya, N., Sugimura, T., Takatsuka, J., et al. (2005). Up-regulation of hnRNP A1 gene in sporadic human colorectal cancers. Int. J. Oncol. 26, 635-640. doi: 10.3892/ijo.26.3.635

Wen, W., Huang, J. Y., Bao, T., Zhou, J., Xia, H. X., Zhang, X. H., et al. (2016). Increased electrocatalyzed performance through hairpin oligonucleotide aptamer-functionalized gold nanorods labels and graphene-streptavidin nanomatrix: highly selective and sensitive electrochemical biosensor of carcinoembryonic antigen. Biosens. Bioelectron. 83, 142-148. doi: 10.1016/j. bios.2016.04.039

Xodo, L., Paramasivam, M., Membrino, A., and Cogoi, S. (2008). Protein hnRNPA1 binds to a critical G-rich element of KRAS and unwinds G-quadruplex structures: implications in transcription. Nucleic Acids Symp. Ser. 52, 159-160. doi: 10.1093/nass/nrn081

Xu, R. M., Jokhan, L., Cheng, X. D., Mayeda, A., and Krainer, A. R. (1997). Crystal structure of human UP1, the domain of hnRNP A1 that contains two RNA-recognition motifs. Structure 5, 559-570. doi: 10.1016/S0969-2126(97)00 211-6

Yang, C., Kulkarni, M., Lim, M., and Pak, Y. (2017). Insilico direct folding of thrombin-binding aptamer G-quadruplex at all-atom level. Nucleic Acids Res. 45, 12648-12656. doi: 10.1093/nar/gkx1079

Zhang, J., Kong, L., Guo, S., Bu, M., Guo, Q., Xiong, Y., et al. (2017). hnRNPs and ELAVL1 cooperate with uORFs to inhibit protein translation. Nucleic Acids Res. 45, 2849-2864. doi: 10.1093/nar/gkw991

Zhang, Q. S., Manche, L., Xu, R. M., and Krainer, A. R. (2006). hnRNP A1 associates with telomere ends and stimulates telomerase activity. RNA 12, 1116-1128. doi: 10.1261/rna.58806

Zhou, Y., Yu, Y., Gao, L., Fei, Y., Ye, T., Li, Q., et al. (2018). Structuring polarityinverted TBA to G-quadruplex for selective recognition of planarity of natural isoquinoline alkaloids. Analyst 143, 4907-4914. doi: 10.1039/C8AN01561A

Conflict of Interest: The authors declare that the research was conducted in the absence of any commercial or financial relationships that could be construed as a potential conflict of interest.

Copyright (C) 2021 Wu, Wang, Li, Zhang, Yang, Ma, Guan and Yang. This is an open-access article distributed under the terms of the Creative Commons Attribution License (CC BY). The use, distribution or reproduction in other forums is permitted, provided the original author(s) and the copyright owner(s) are credited and that the original publication in this journal is cited, in accordance with accepted academic practice. No use, distribution or reproduction is permitted which does not comply with these terms. 